\title{
低共熔溶剂在绿色有机合成中的应用
}

\author{
熊兴泉* 韩骞石霖 肖上运毕成 \\ (华侨大学材料科学与工程学院 福建省高校功能材料重点实验室 厦门 361021)
}

\begin{abstract}
摘要 自 1991 年 “绿色化学” 的概念被提出以来, 溶剂的绿色化一直备受关注. 在过去的二十年时间里, 离子液体得 到长足发展, 但是随着研究的不断深入, 离子液体的部分弊端也慢慢浮现. 在最近几年兴起了一种新型的低熔点混合 物溶剂一一低共熔溶剂. 与传统离子液体相比，低共熔溶剂制备简单、价格便宜、均具有生物可降解性，因此被用来作 为一种新型绿色溶剂. 近年来, 低共熔溶剂在绿色有机合成中的应用受到了越来越多的应用. 本文重点从低共熔溶剂 在环化反应、取代反应、加成反应、多组分反应以及酶催化反应等方面的应用讨论近几年来低共熔溶剂的研究进展，并 对其发展趋势进行展望.
\end{abstract}

关键词＼cjkstart低共熔溶剂; 绿色化学; 有机合成; 催化

\section{Application of Deep-Eutectic Solvents in Green Organic Synthesis}

\author{
Xiong, Xingquan* Han, Qian Shi, Lin Xiao, Shangyun Bi, Cheng \\ (Key Laboratory for Functional Materials of Fujian Higher Education, College of Materials Science and Engineering, \\ Huaqiao University, Xiamen 361021)
}

\begin{abstract}
Since the definition of "green chemistry" was proposed in 1991, the green solvents have attracted considerable attention. In the two decades ago, ionic liquids grew up. However, as following the research, it was found that ionic liquids had some drawbacks. Nowadays, some new types of low-transition-temperature mixtures, i.e. deep-eutectic solvents (DES) are raised. Compared with the traditional ionic liquids, deep-eutectic solvents are greener, cheaper and more accessible. Furthermore, the preparation methods of deep-eutectic solvents are easy, and the components of them are biodegradable. In recent years, deep-eutectic solvents have been applied as green solvents in organic synthesis widely. In this review, the application of deep-eutectic solvents in cyclization reactions, replacement reactions, addition reactions, multicomponent reactions, enzyme catalytic reactions and other reactions in the last few years are briefly summarized, and the prospects of DESs are also discussed.
\end{abstract}

Keywords deep-eutectic solvents; green chemistry; organic synthesis; catalysis

室温离子液体(RTIL)在近二十年来备受关注, 特别 是在化学合成、材料化学、电化学领域以及最近兴起的 细胞质的预处理领域 ${ }^{[14]} .2009$ 年 Seddon 和同事 ${ }^{[5]}$ 就曾 报道，现有的许多离子液体已用于工业生产，并且有 250 种被商品化. 但由于离子液体对于杂质的存在十分 敏感，即使微量的杂质存在也有可能影响其理化性质， 为此离子液体的制备过程要求极其高, 这也就导致了离 子液体其价格居高不下, 从而限制了其在工业中的广泛
应用. 同时, 离子液体存在毒性和生物降解性差等缺点. 针对这些弊端，科研工作者发展了一种新型、绿色 的低熔点混合物溶剂，也就是低共熔溶剂(DESs)作为有 机反应的溶剂 ${ }^{[6 ~ 8]}$. 与传统离子液体相比, 低共熔溶剂 制备简单、价格便宜、均具有生物可降解性，因此被用 来作为一种新型绿色溶剂. 低共熔溶剂是由氢键受体 (HBA)(季铵盐)与氢键供体(HBD)(羧酸、多元醇、尿素 等)组成或由季铵盐与金属盐类物质(如 $\mathrm{ChCl} / \mathrm{MCl}_{2}$ )组

\footnotetext{
*E-mail: xxqluli@hqu.edu.cn

Received August 5, 2015; revised October 31, 2015; published online December 4, 2015.

Project supported by the National Natural Science Foundation of China (No. 21004024), the Natural Science Foundation of Fujian Province (No. 2016J01063), the Program for New Century Excellent Talents in Fujian Province (No. 2012FJ-NCET-ZR03), the University Distinguished Young Research Talent Training Program of Fujian province (No. 11FJPY02) and the Promotion Program for Young and Middle-aged Teacher in Science and Technology Research of Huaqiao University (No. ZQN-YX103).

国家自然科学基金(No. 21004024)、福建省自然科学基金(No. 2016J01063)、福建省高校新世纪优秀人才支持计划(No. 2012FJ-NCET-ZR03)和福建省 高校杰出青年科研人才培育计划(No. 11FJPY02)以及华侨大学中青年教师科研提升资助计划(No. ZQN-YX103)资助项目.
} 
成, 并且组成低共熔溶剂的物质大多都是可以再生的. 虽然之前有一部分人认为低共熔溶剂是离子液体的一 种, 但现在看来两者还有本质上的区别的: (1)低共熔溶 剂 并不是由完全离子化物质组成; (2)其键合形式也并 非是离子键, 而是以氢键为主. 虽然在此之前也有关于 低共熔溶剂的综述报道, 但是鲜有针对最近几年其在有 机领域的最新应用研究的报道. 为此, 本文重点从低共 熔溶剂在环化反应、取代反应、加成反应、多组分反应 以及酶催化反应等方面的应用讨论近几年来低共熔溶 剂的研究进展, 并对其发展趋势进行展望.

\section{1 简介低共熔溶剂}

低共熔溶剂是由 Abbott 等 ${ }^{[9]}$ 在 2003 年发表的文章 中首次提出, 他们通过氯化胆碱 $\left(\mathrm{ChCl} ; \mathrm{m}\right.$.p. $\left.302{ }^{\circ} \mathrm{C}\right)$ 与 尿素(urea; m.p. $113{ }^{\circ} \mathrm{C}$ )以物质的量比为 $2: 1$ 共混形成 了一种低于两种组成物质熔点的混合物 (m.p. $12{ }^{\circ} \mathrm{C}$ ), 这种混合物也是第一种被定义为低共熔溶剂的物质. 而 随着研究的不断深入, 诸多学者都提出了自己的见解. 如 Abbott 等提出了对于熔点降低的见解: 氢键供体(尿 素)与季铵盐 $(\mathrm{ChCl})$ 之间形成氢键作用(Scheme 1), 从而 抑制了固体的析出. 这种由 $\mathrm{ChCl}$ 组成的低共熔溶剂有 着许多的优点: (1)价格低廉; (2)水的化学惰性(易于储 存); (3)易于制备, 低共熔溶剂通常情况条件下是由两种 及以上便宜安全无毒的物质经过简单的混合而制得的; (4)大部分低共熔溶剂是可生物降解的, 并且有良好的 生物相容性和无毒性, 增强了介质的绿色度. 同时, 由 于低共熔溶剂的理化性质(密度、粘度、折射率、导电 性、表面张力以及化学惰性等)与常见的离子液体相近, 因此低共熔溶剂是一种有望能够代替离子液体的溶剂. 就目前公开发表的文献来看, 常见的 HBD 与 HBA 如图 1 所示.

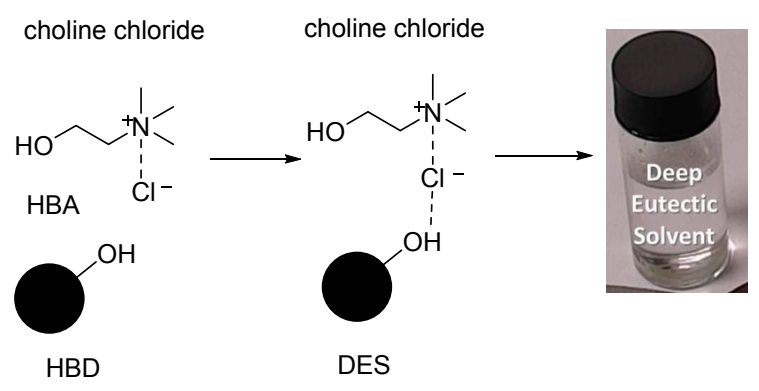

图式 1 低共熔溶剂的制备

Scheme 1 Preparation of deep-eutectic solvent

\section{2 低共熔溶剂在有机合成中的应用}

在有机合成领域, 由于经常使用的常规有机溶剂存 在有潜在毒性、安全性以及不价廉易得等问题, 合适溶
剂的选择是非常重要的, 所以溶剂的绿色化一直是研究 的热点. 溶剂不仅要能溶解各组分反应物, 更重要的是 能使催化剂与各组分反应物之间紧密接触, 以及在反应 完成之后能够便于催化剂的回收和生成物的分离纯化. 因此，对于一个有机反应来说，绿色、价廉以及合适的 溶剂的选择成为了一个重要的研究课题. 近几年, 由于 对离子液体研究的进一步深入, 其在稳定纳米粒子、固 载均相催化剂和催化转化再生材料等方面有着明显的 优势. 虽然离子液体存在着诸多的优点, 但是其也有明 显缺陷, 即制备离子液体的过程不绿色、需要多步合成 导致其不价廉易得等. 这些问题得不到解决，其发展必 定受到限制. 然而低共熔溶剂的出现为溶剂的绿色化、 廉价化的研究提供了一个新的方向. 下面将结合实例对 其在绿色有机合成中的应用进行阐述.

\section{1 低共熔溶剂在环化反应中的应用}

2002 年 Davies 等 ${ }^{[10]}$ 报道了 Diels-Alder 反应在 $\mathrm{ChCl} /$ $\mathrm{MCl}_{2}$ 中进行的可行性. 实验结果表明, 路易斯酸性的低 共熔溶剂能显著地增加反应的速率, 各种二烯和亲二烯 体在 $\mathrm{ChCl} / \mathrm{MCl}_{2}$ 的进行 Diels-Alder 反应(Scheme 2), 其 产率在 $85 \% \sim 91 \%$, 而内/外选择性在 $83: 17$ 到 $97: 3$ 的范围内.

2005 年, Imperato 等 ${ }^{[1]}$ 研究了在不同碳水化合物, 如果糖、麦芽糖、乳糖、甘露醇、葡萄糖和山梨醇与尿 素和 $N, N$-二甲基嫝(DMU)两两组合而成的系列低共熔 溶剂中, Diels-Alder 反应的差异性. 研究表明, 在果糖/ DMU (7/3)的低共熔溶剂中, Diels-Alder 反应在 $71{ }^{\circ} \mathrm{C}$ 条件下反应 $8 \mathrm{~h}$ 后获得了比较满意的效果(Eq. 1). 作者 还采用其他的一些低共熔溶剂 用于该反应中, 其产率 在 72\% 100\%之间，而其产物的内/外选择性比在 2.7/1 到 $5 / 1$ 之间. 相较而言, 基于碳水化合物的低共熔溶剂 一点也不逊色于其他绿色溶剂(例如超临界 $\mathrm{CO}_{2}$ 、水、咪 唑类离子液体等).

在 2009 年, König 和同事 ${ }^{[12]}$ 报道了在含有 $5 \%$ 脯氨 酸的 $L$-肉碱/尿素 $(2: 3)$ 组成的低共熔溶剂体系中进行 催化 Diels-Alder 反应. 当反应在 $80{ }^{\circ} \mathrm{C}$ 进行 $4 \mathrm{~h}$ 后, Diels-Alder 的加成产物的产率高达 93\% (Eq. 2). 该 低 共熔溶剂体系可以替代了一些溶剂，如离子液体、超临 界二氧化碳、水等, 而且脯氨酸可以代替 $\operatorname{Sc}(\mathrm{OTf})_{3}$ 作为 催化剂, 避免了钪金属离子盐对产物的污染.

Shankarling 等 ${ }^{[13]}$ 报道了通过低共熔溶剂 $(\mathrm{ChCl} / \mathrm{urea})$ 作为溶剂应用超声和热辅助合成氨基噁唑衍 生物(Eq. 3). 结果表明, 应用超声辅助方法在 $8 \mathrm{~min}$ 内 合成的目标产物产率为 $90 \%$, 这要远远高于应用普通热 辅助方法合成 $3.5 \mathrm{~h}$ 生成的产率(其产率为 $69 \%$ ). 应用超 


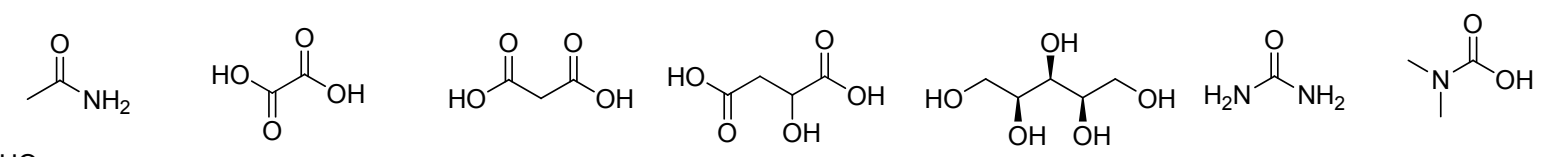

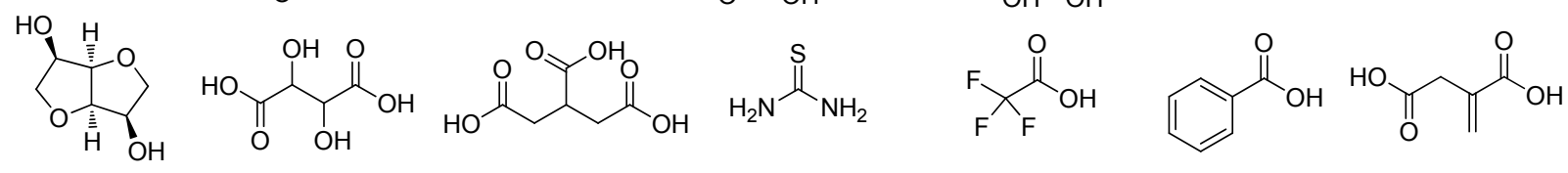
$\underbrace{\mathrm{OHH}_{\mathrm{N}}^{\mathrm{O}_{\mathrm{O}}}}_{\mathrm{OH}} \sum_{\mathrm{N}}^{\mathrm{HN}}$ $\mathrm{NH}_{\mathrm{NH}}^{\mathrm{N}}$ (N) $\mathrm{Cll}_{\mathrm{OH}}^{\mathrm{O}}$<smiles>CCCCCCCC/C=C/CCCCCCCC(=O)O</smiles>
$\prod_{\mathrm{O}}^{\mathrm{O}}$<smiles>CCCCCC=CCC=CCCCCCCCC(=O)O</smiles><smiles>CCCCCCCCCC(=O)O</smiles>

氢键供体

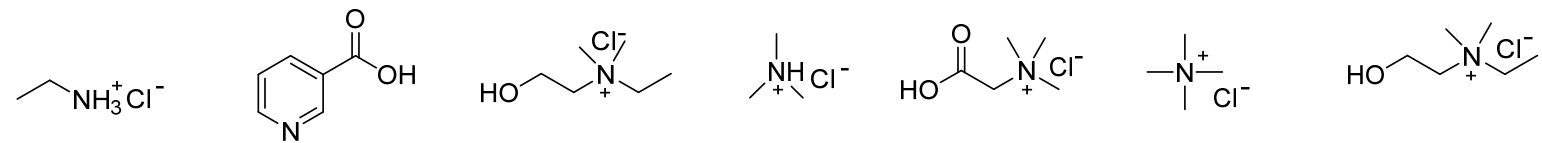

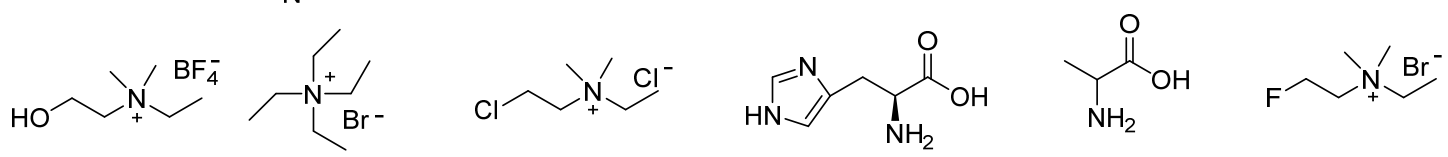

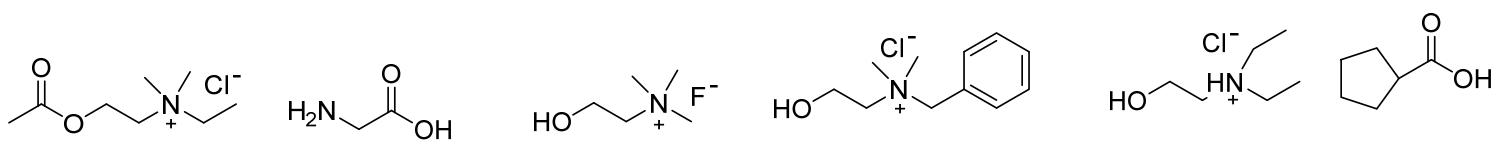<smiles>CCN(CC)CC(=O)Nc1c(C)cccc1C</smiles><smiles>[Cl+]P(c1ccccc1)(c1ccccc1)(c1ccccc1)c1ccccc1</smiles><smiles>Br[P-](Cc1ccccc1)(c1ccccc1)c1ccccc1</smiles><smiles>CCCCN(CCCC)CCCC</smiles>

氢键受体

Figure 1 Typical structures of HBDs and HBAs for deep-eutectic solvent synthesis 


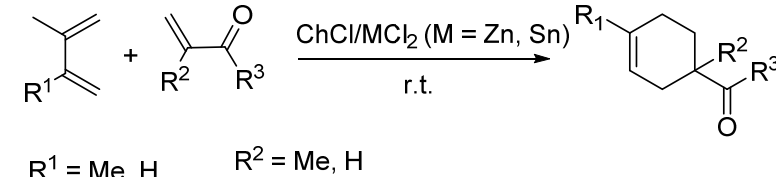

$$
\mathrm{R}^{1}=\mathrm{Me}, \mathrm{H} \quad \mathrm{R}^{3}=\mathrm{H}, \mathrm{Me}, \mathrm{OMe}
$$

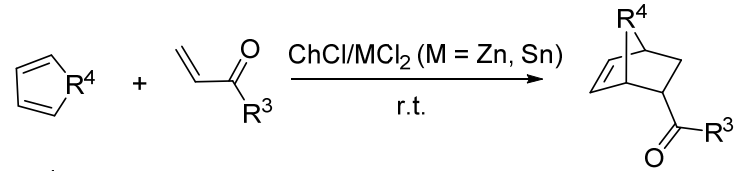

$\mathrm{R}^{4}=\mathrm{CH}_{2},\left(\mathrm{CH}_{2}\right)_{2}$

图式 2 在低共熔溶剂体系中进行 Diels-Alder 反应

Scheme 2 Diels-Alder reaction in deep-eutectic solvent

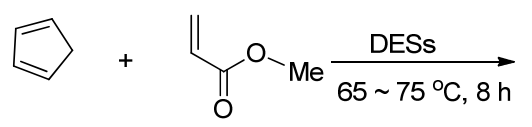<smiles>COC(=O)C1C2C=CC(C2)C1C(C)=O</smiles>

exolendo $=2.9 / 1$

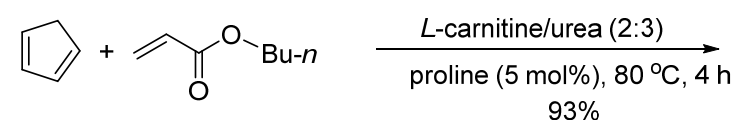
$93 \%$

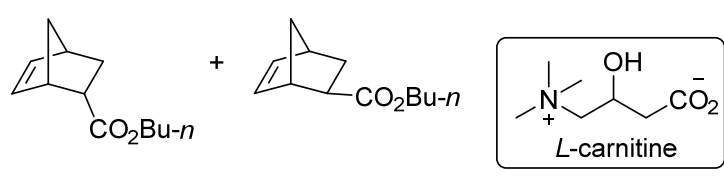

exolendo $=3.5 / 1$<smiles>[R]c1ccc(C(=O)CBr)cc1</smiles>
$\underset{\text { DES }, 65^{\circ} \mathrm{C}}{\stackrel{\text { oES, ultrasound, } 35^{\circ} \mathrm{C}}{\longrightarrow}}$<smiles>[R]c1ccc(-c2coc(N)n2)cc1</smiles>

$\mathrm{R}=\mathrm{H}, \mathrm{Br}, \mathrm{NO}_{2}, \mathrm{OCH}_{3}$

声合成产物的结晶度 $21.12 \%$, 相较于应用普通热方法 合成产率的结晶度 $8.33 \%$, 结晶度增加了近 $60 \%$ 左右. 除此之外, 通过计算超声合成方法要比普通热合成方法 节约近 70\%的能量.

2012 年, Zhang 等 ${ }^{[14]}$ 报道了通过 2-氨基苯丙酮、乙 醛和乙酸铵在碳水化合物基的低共熔溶剂中合成喹唑 啉的衍生物(Eq. 4). 特别是在麦芽糖/DMU/NH${ }_{4} \mathrm{Cl}$ 中, 喹唑啉的产率高达 $92 \%$. 这中合成方法底物来源范围 广, 并且产物可以通过结晶或者用乙酸乙酯从低共熔溶 剂中分离, 这样低共熔溶剂就可以再次循环使用了.<smiles>Nc1ccccc1C(=O)c1ccccc1</smiles>

\section{2 低共熔溶剂在取代反应中的应用}

2006 年, König 等 ${ }^{[10]}$ 研究了苯基硼酸与芳基溴在不 同碳水化合物-尿素-无机盐的组成的低共熔溶剂中钯 催化下的 Suzuki 偶联反应. 结果表明, 在该体系中 Suzuki 偶联反应能够顺利进行，联芳基产物分离后的产 率为 $78 \%$ \% $98 \%$. 随后，该小组又把钯催化的 Heck 反应 成功在 $D$-甘露糖/DMU (3/7)体系中进行. 在均相催化剂 [例如 $\mathrm{Pd}(\mathrm{OAc})_{2} 、 \mathrm{PdCl}_{2}\left(\mathrm{PPh}_{3}\right)_{2}$ ]条件下，交联产物的产率 十分理想 $(77 \% \sim 91 \%)$ (Eq. 5). 相较于传统的咪唑类离 子液体, 在低共熔溶剂中进行该反应的反应温度更低 $\left(80{ }^{\circ} \mathrm{C}\right.$ vs. $\left.120{ }^{\circ} \mathrm{C}\right)$, 反应速率更快. 当作者使用异相催 化剂(例如 $\mathrm{Pd} / \mathrm{C}$ ), 发现该反应反应速率有明显下降, 这 归结于异相催化剂的加入增加了体系的粘度, 体系中的 物质运动受阻.

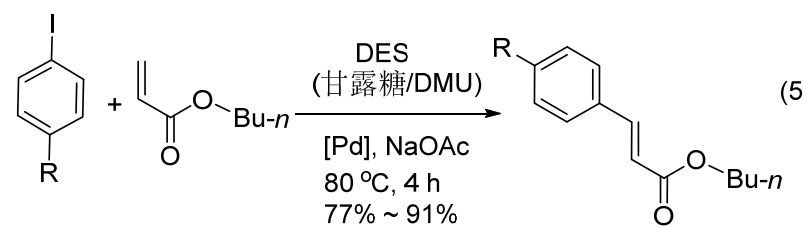

2009 年, Zou 等 ${ }^{[15]}$ 报道了在 $\mathrm{ChCl} /$ 对甲苯磺酸 $(p-\mathrm{TsOH})$ (比例为 $1: 1)$ 的低共熔溶剂体系中合成 $\alpha, \alpha$-二 氯苯甲酩的方法. 在低共熔溶剂与苯乙酮的比率为 1 : 1 的体系中, 加入作为氯源的 1,2-二氯-5,5-二甲基乙内 酰嫝，在室温下 $\alpha, \alpha$-二氯化取代物的产率为 $86 \%$ (Eq. 6). 在反应结束后, $\alpha, \alpha$ 二氯化取代物在 $\mathrm{ChCl} / p-\mathrm{TsOH}$ 的低 共熔溶剂中应用甲基叔丁基醚作为分离溶剂, 产物很容 易从低共熔溶剂体系中分离出来, 为此作者成功地将该 低共熔溶剂回收了 5 次.

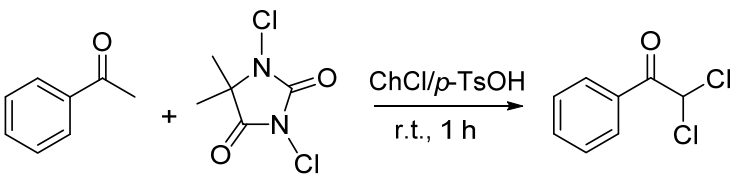

在 2010 年, Shankarling 等 ${ }^{[16]}$ 研究了 1-氨基葱-9,10醌衍生物在 $\mathrm{ChCl}$ 和尿素的体系中进行亲电取代反应. $80{ }^{\circ} \mathrm{C}$ 以及使用 2.5 equiv.液溴的反应条件下，二溴取代 
产物的产率为 $84 \% \sim 95 \%$ (Eq. 7), 相较于传统的有机溶 剂(甲醇, 氯仿), 反应条件更为简单, 反应速率更高, 产 率亦有所增高, 而且避免了有毒溶剂与催化剂的使用, 为其绿色合成提供了新的思路.

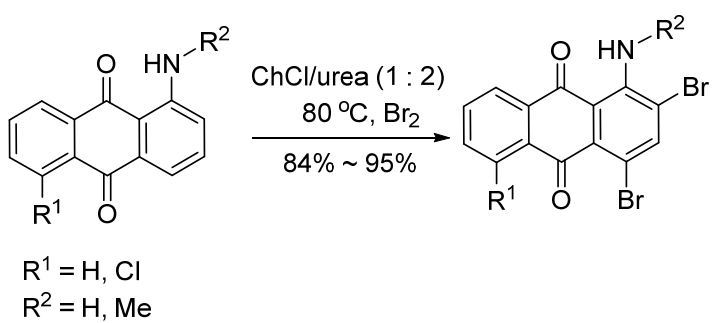

2011 年 Shankarling 等 ${ }^{[17]}$ 报道了 $\mathrm{ChCl} /$ 尿素和 $\mathrm{ChCl} /$ 甘油的低共熔溶剂可以用于制备单取代 $N$-芳烃胺的催 化剂和溶剂(Eq. 7). 特别值得注意的是, 由 $\mathrm{ChCl} /$ 尿素组 成的低共熔溶剂要比由 $\mathrm{ChCl} /$ 甘油的低共熔溶剂的效果 明显, 其原因可能是前者的碱性更强. 这个方法有着很 强的应用前景, 这是由于芳胺和烷基溴化物的种类繁 多, 在温度达到 $50{ }^{\circ} \mathrm{C}$ 时相应的单取代 $N$-芳烃胺的产率 为 $70 \% \sim 89 \%$. 在反应结束后, 产物可以通过乙酸乙酯 从由 $\mathrm{ChCl} /$ 尿素组成的低共熔溶剂中萃取分离出来. 最 后作者还报道了其成功将低共熔溶剂回收 5 次, 并且 反应产率没有明显下降, 从而克服了酶催化反应过程中 由于循环使用而导致酶失活的缺陷.

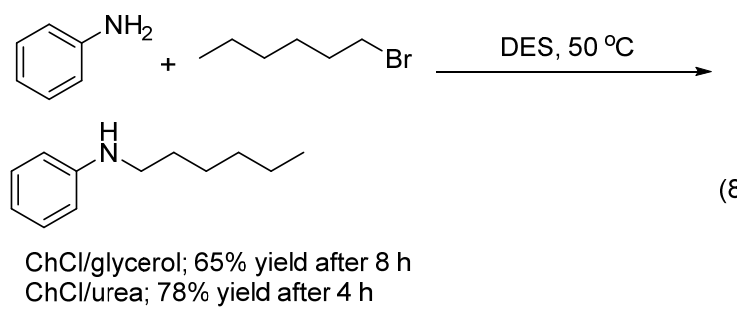

2012 年, Germani 等 ${ }^{[18]}$ 报道了应用新型布朗斯特酸 低共熔溶剂作为羧酸和醇的酯化媒介. 他们报道的新 型布朗斯特酸低共熔溶剂中不含有任何的卤素和金属 原子, 它的组成是季铵盐与甲基磺酸盐或对苯甲磺酸 盐, 其中甲基磺酸盐与对苯甲磺酸盐作为季铵盐的反离 子存在, 这样就避开了一些弊端. 结果表明, 应用低共 熔溶剂作为媒介进行羧酸和醇的酯化反应其反应条件 温和(Eq. 9), 并且产率大部分高于 $70 \%$. 对低共熔溶剂 进行了循环次数的测定发现, 当低共熔溶剂循环 8 次后 仍然具有催化效果, 并且其产率高达 $92 \%$.

Imperato 等 ${ }^{[19]}$ 研究了在各种碳水化合物-尿素-盐 熔体中 Stille 交联反应. 研究表明, 在 $90{ }^{\circ} \mathrm{C}$ 以及四乙基 锡或四丁基锡等存在条件下进行交联反应(Scheme 3), 麦芽糖/DMU/ $\mathrm{NH}_{4} \mathrm{Cl}$ 组成的低共熔溶剂能使锡上的丁基
和乙基很好地转移到碘代苯上. 而后作者又使用尿素代 替 $\mathrm{DMU}$, 产率没有明显下降. 但相较于之前的反应条 件, 成本更低、更为绿色. 值得一提的是, 与咪唑类离子 液体相比，该体系没有联苯产物的生成. 另外，当在多 元醇/ $\mathrm{DMU} / \mathrm{NH}_{4} \mathrm{Cl}$ (多元醇是乳糖、麦芽糖、甘露醇、山 梨醇)混合体系组成的低共熔溶剂中，应用 Stille 交联偶 合通过芳基溴化物与三丁基锡制备联苯，反应完成后， 目标产物的产率在 $85 \% \sim 100 \%$. 更为重要的是, 钯催化 剂可回收再循环使用. 另外, 由于产物在加入水的催化 相中是不相溶的, 因此相较于二甲基甲酰胺、二氧六环 而言, 这种分离方法是更为绿色和环境友好.
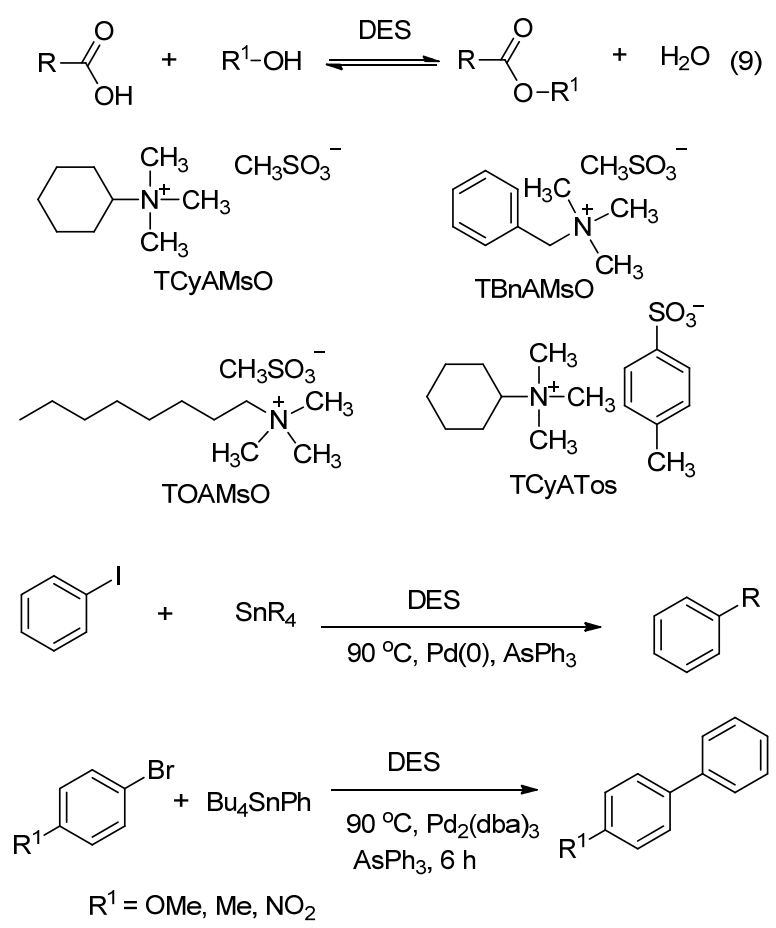

图式 3 在低共熔溶剂体系中进行钯催化的 Stille 偶联反应 Scheme 3 Palladium-catalyzed Stille coupling reaction in deepeutectic solvent

2015 年, Wang 等 ${ }^{[20]}$ 研究了在不同的低共熔溶剂, 如 $\mathrm{ChCl} / \mathrm{ZnCl}_{2} 、 \mathrm{Me}_{4} \mathrm{NCl} / \mathrm{ZnCl}_{2}$ 等体系中，以芳香醛或脂 肪醛、富电子的烯烃为原料, 在 $60 \sim 100{ }^{\circ} \mathrm{C}$ 加热的条件 下，最高以 $94 \%$ 的产率进行 Friedel-Crafts 烷基化反应， 制备出相应的烷基化的芳香族化合物. 结果表明, 在对 模型反应进行研究时, 由于低共熔溶剂体系较为稳定, 该催化体系在循环 5 次后 Friedel-Crafts 烷基化反应产率 还能达到 $89 \%$. 该反应体系反应过程简单易行、循环次 数较多且每次产率降低不明显.

\section{3 低共熔溶剂在加成反应中的应用}

2011 年, Shankarling 等 ${ }^{[21]}$ 报道了在 $\mathrm{ChCl} /$ 尿素(1： 2)的低共熔溶剂中通过碱催化的 Perlin 反应合成肉桂酸 
(Eq. 10). 由于低共熔溶剂本身就显碱性, 所以由 $\mathrm{ChCl} /$ 尿素组成的低共熔溶剂具有促使反应进行的特性. 反应 物安息香酸的衍生物与乙酸䣶等物质的量地添加在 $\mathrm{ChCl} /$ 尿素组成的低共熔溶剂中并加热至 $30{ }^{\circ} \mathrm{C}$, 之后产 生的一系列的肉桂酸衍生物，都有良好的产率 $(62 \%$ $92 \%)$. 相较于传统的合成方法 $[V$ (苯甲醛)：V(乙酸酎)： $V($ 三水合醋酸钠 $\left.)=1: 2: 3,40{ }^{\circ} \mathrm{C}\right)$ 而言, 原料更为节 约, 反应温度更低, 能耗更小.

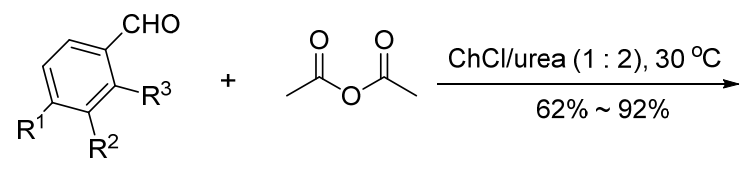

$$
\begin{array}{ll}
\mathrm{R}^{1}=\mathrm{H}, \mathrm{Cl}, \mathrm{CN} \\
\mathrm{R}^{2}=\mathrm{H}, \mathrm{Cl}, \mathrm{CF}_{3}, \mathrm{OMe} \\
\mathrm{R}^{3}=\mathrm{H}, \mathrm{Cl}, \mathrm{OMe}
\end{array}
$$

随后，该小组 ${ }^{[2]}$ 还报道了应用 $\mathrm{ChCl}$ 基低共熔溶剂 催化 $\mathrm{C}-\mathrm{C}$ 偶联反应制备 $\beta$-羊基功能化的衍生物. $\beta$-羊圣 基功能化衍生物是制备硝基烯烃、2-氨基醇、2-硝基酮 等物质的中间产物. 作者应用 $\mathrm{ChCl}$ 基低共熔溶剂催化 芳醛和硝基甲烷、乙腈、乙酸之间的 $\mathrm{C}-\mathrm{C}$ 偶联反应 (Scheme 4). 研究表明, 反应很短的时间内有很可观的 反应产率. 而且低共熔溶剂循环四次后其催化效果仍然 很高(产率高达 90\%).

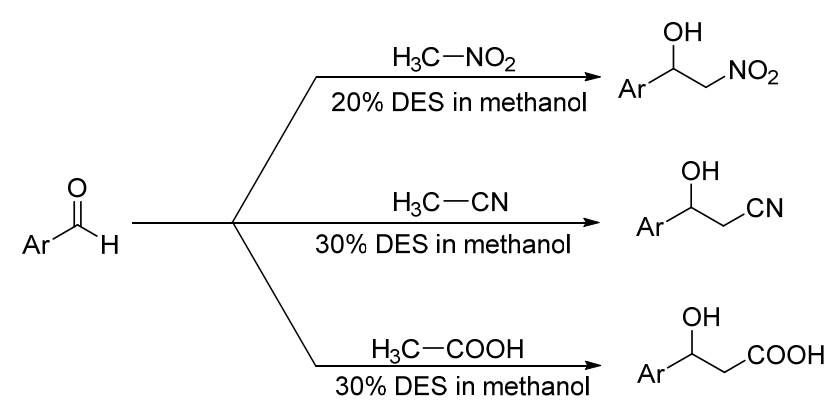

图式 4 在低共熔溶剂中合成 $\beta$-羟基衍生物

Scheme 4 Synthesis of $\beta$-hydroxy derivatives using deep-eutectic solvent

由于 $\mathrm{ChCl} /$ 尿素组成的低共熔溶剂是碱性的, 除此 之外它还能催化的反应有 Knoevenagel 反应. Sonawane 等 ${ }^{[23]}$ 报道了在 $\mathrm{ChCl} /$ 尿素 $(1: 2)$ 的低共熔溶剂中合成具 有共轭结构的二苯氨的衍生物. 值得注意的是, 在以碱 性的 $\mathrm{ChCl} /$ 尿素组成的低共熔溶剂体系中, 其产物产率 最高可达 $95 \%$, 该方法的成功尝试为合成共轭结构的二 苯氨的衍生物提供了一个可以替代应用脂肪酶和咪唑 类离子液体的合成路线(Scheme 5). 结果表明, 由于低 共熔溶剂与反应产物易于分离, 所以低共熔溶剂被成功 地回收并重复使用了五次.
2012 年, Azizi 等 ${ }^{[24]}$ 报道了应用 $\mathrm{ChCl} /$ 尿素组成的低 共熔溶剂制备二硫代氨基甲酸酯的方法. 结果表明, 采 用低共熔溶剂作为绿色反应溶剂, 缩短了反应时间, 降 低了反应的成本，提高了反应的产率，并且反应没有有 机溶剂的参与, 这就为二硫代氨基甲酸酯衍生物的制备 提供了一个种环境友好的合成路线(Eq. 11).

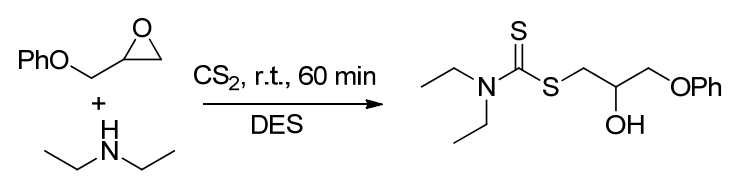

最近, Azizi 等 ${ }^{[25]}$ 报道, 在 $\mathrm{ChCl} /$ urea $(1: 2)$ 组成的低 共熔溶剂中, 以醛或环氧化合物与氭化三甲基硅烷为起 始原料, 使用纳米级的磁性 $\mathrm{Fe}_{3} \mathrm{O}_{4}$ 作为催化剂, 在 $60{ }^{\circ} \mathrm{C}$ 加热的条件下反应 $30 \sim 200 \mathrm{~min}$, 可以以最高为 $92 \%$ 的产率制备出 $\alpha$-羟基腈或 $\beta$-羟基腈. 研究表明, 由 于 $\mathrm{Fe}_{3} \mathrm{O}_{4}$ 纳米催化剂具有磁响应的性质, 因此可以在外 加磁铁的作用下顺利对其进行回收再使用. 由于 $\mathrm{Fe}_{3} \mathrm{O}_{4} / \mathrm{DES}$ 较为稳定, 该催化剂在循环 4 次后产率还能 达到 $85 \%$. 该催化体系不仅适用于芳香醛和脂肪醛，同 样也对芳香族环氧化合物和脂肪族环氧化合物有很好 的催化效果. 该反应体系最大的优势是反应时间短，反 应条件温和以及过程简单且绿色.

\section{4 低共熔溶剂在多组分反应中的应用}

$\mathrm{CuAAC}$ 反应在有机合成中是一种非常有用的合成 工具, 广泛地应用于药物制备、核苷衍生物的制备、聚 合物化学以及超分子材料等领域 ${ }^{[26]}$. 由于 $D$-山梨醇/尿 素 $/ \mathrm{NH}_{4} \mathrm{Cl}(7: 2: 1)$ 组成的低共熔溶剂具有很稳定的性 质, 所以可以作为像叠氮这样强亲核试剂的溶剂. 在 $5 \% \mathrm{CuI}$ 中 $85{ }^{\circ} \mathrm{C}$ 反应 $5 \mathrm{~h}$ 后 1,4-取代-1,2,3-三唑的产率 为 $93 \%$ (Scheme 5). 更重要的是可以使叠氮化与环加成 在一锅中完成 ${ }^{[10]}$.

2014 年, Azizi 等 ${ }^{[27]}$ 报道了通过水杨醛、丙二腈以及 不同的亲核试剂在低共熔溶剂( $\mathrm{ChCl} / \mathrm{urea})$ 中合成为相应 的苯并吡喃及其衍生物(Eq. 12). 该方法采用无毒、成本 低、对环境友好的 低共熔溶剂 作为溶剂, 并且该反应 无需催化剂, 反应后处理简单, 普适性好, 产率高(普遍 高于 $80 \%$ )等特点.

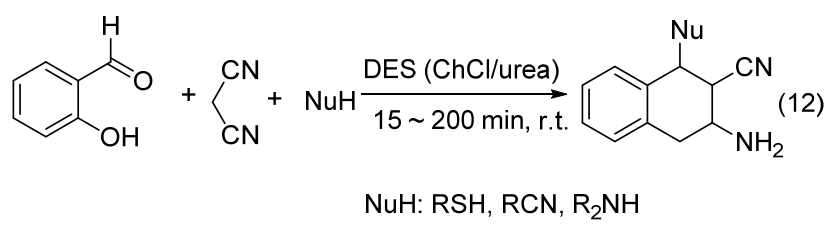




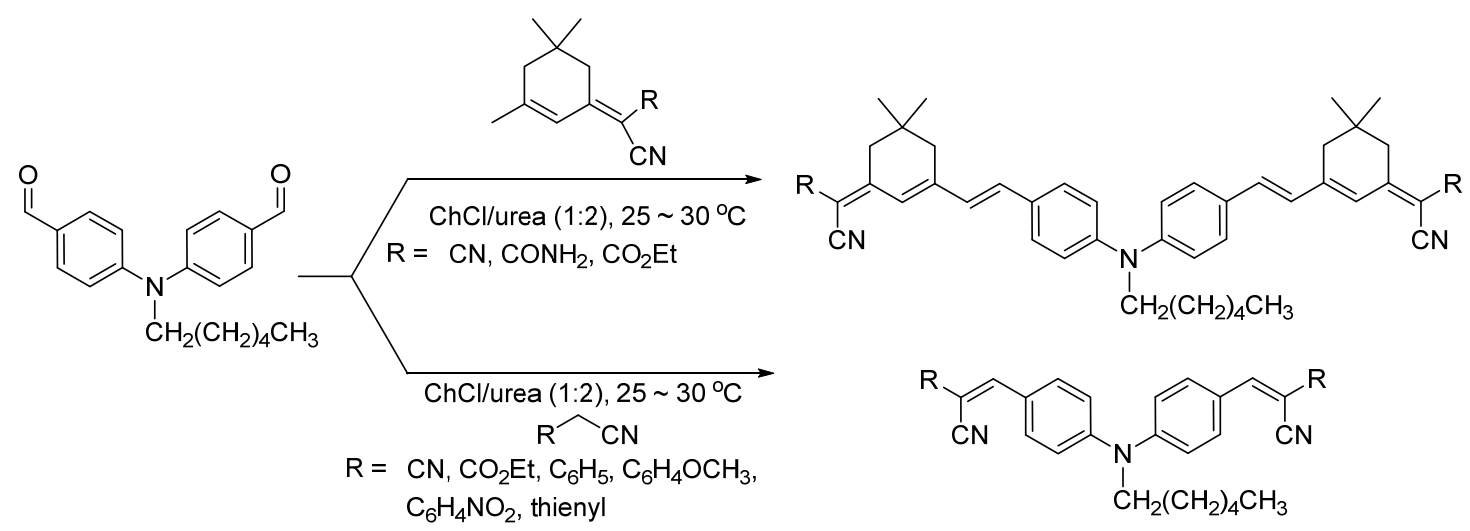

图式 5 在低共熔溶剂体系中碱催化的 Knovenagel 反应

Scheme 5 Base-catalyzed Knovenagel reaction in deep-eutectic solvent

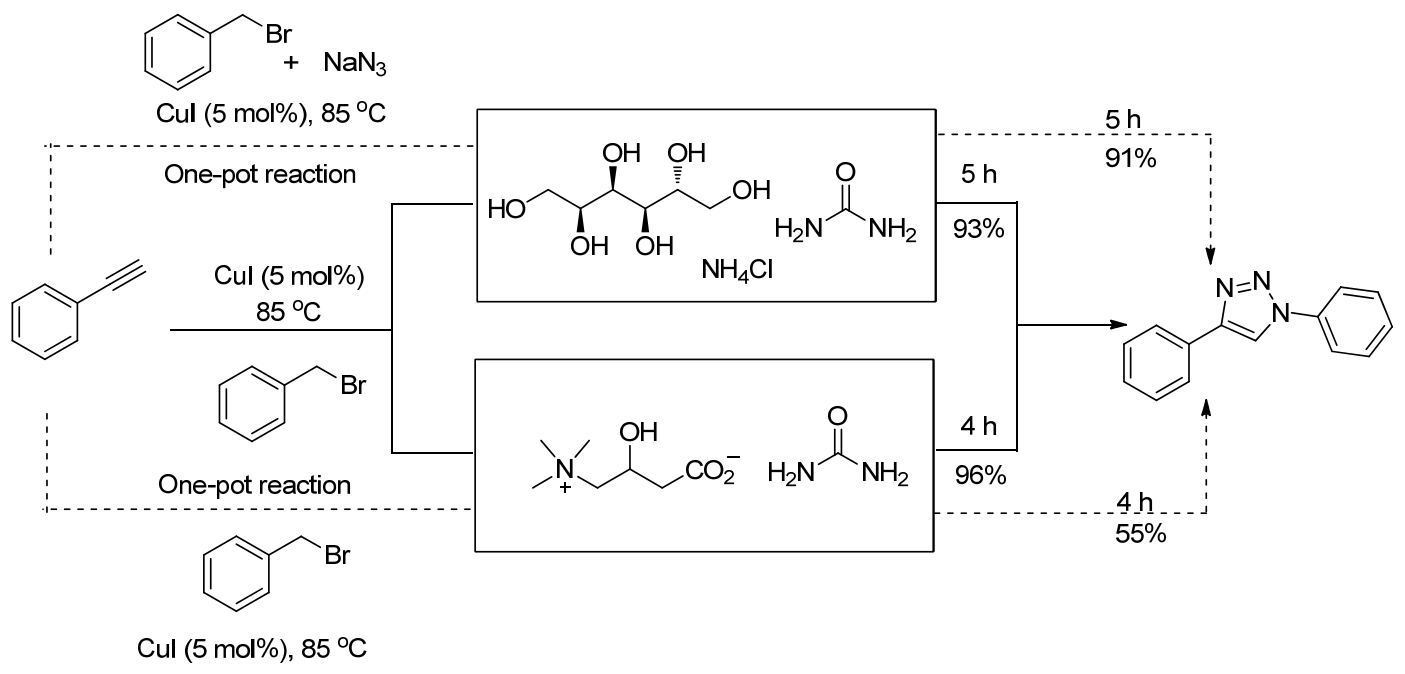

图式 6 在低共熔溶剂体系中进行 $\mathrm{CuAAC}$ 反应

Scheme 6 CuAAc reaction in deep-eutectic solvent

最近, Keshavarzipour 等 ${ }^{[28]}$ 报道了在 $\mathrm{ChCl} / \mathrm{ZnCl}_{2}$ 催 化体系中, 在室温条件下系统研究了醛、有机胺、酮(包 括丙酮、苯乙酮类物质)之间的一锅三组分的 Mannich 反应. 研究表明, 在该条件下, 当 $\mathrm{ChCl} / \mathrm{ZnCl}_{2}$ 的用量为 $5 \mathrm{~mol} \%$ 时，可以 $52 \% \sim 98 \%$ 的收率制备出一系列 $\beta$-氨基 羰基化合物. 该课题组进一步研究了 $\mathrm{ChCl} / \mathrm{ZnCl}_{2}$ 的可循 环使用的次数, 发现当 $\mathrm{ChCl} / \mathrm{ZnCl}_{2}$ 循环 4 次后仍然具有 良好的催化效果. 在催化体系 $\mathrm{ChCl} / \mathrm{ZnCl}_{2}$ 中, 实际起催 化作用的是 $\left[\mathrm{ZnCl}_{3}\right]^{-},\left[\mathrm{ZnCl}_{5}\right]^{-}$和 $\left[\mathrm{ZnCl}_{7}\right]^{-}$, 在反应过程 中它们起到 Lewis 碱的作用去活化酮类物质产生相应的 带负电荷的活性中间体.

\section{4 低共熔溶剂在酶催化反应中的应用}

在 2008 年, Kazlauskas 等 ${ }^{[29]}$ 首次报道了在低共熔溶 剂中应用酶催化有机反应. 该课题组在 $\mathrm{ChCl}$ 和尿素组 成的低共熔溶剂中, 以乙酸戊酯和丁醇酯为原料系统研 究了酯与醇之间交换反应酶的活性(Eq. 13). 研究表明,
尽管在 $\mathrm{ChCl}$ 和尿素的水溶液中酶很不稳定, 但是该酶 在 $\mathrm{ChCl} /$ 尿素组成的低共熔溶剂中却很稳定. 在酯交换 反应中, 尽管由于有尿素的存在, 生物酶仍然保持良好 的催化活性.

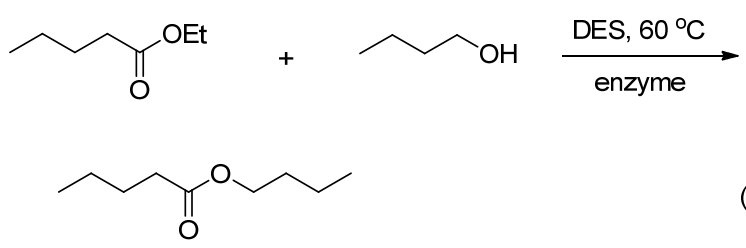

随后, $\mathrm{Zhao}$ 等 ${ }^{[30]}$ 也报道了在 $\mathrm{ChCl}$ 和甘油组成的 低 共熔溶剂 中脂肪酶催化的酯交换反应. 作者首先研究 了山梨酸乙酯和 1-丙醇为反应原料, 在各种低共熔溶剂 体系中和以 Novozym ${ }^{\circledR} 435$ 作为催化剂进行酯交换反应 (Eq. 14). 研究表明, 在 $\mathrm{ChCl} /$ 尿素体系中酶的活性 $(>1$ $\left.\mu \mathrm{mol} \cdot \mathrm{min}^{-1} \cdot \mathrm{g}^{-1}\right)$ 和选择性 $(>99 \%)$ 比较高. 


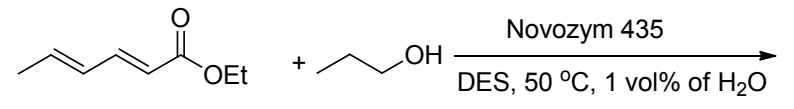<smiles>C/C=C/C=C/C(=O)OCCC</smiles>

另外, 该课题组还研究了在胆碱基质的低共熔溶剂 中生物柴油的合成方法. 生物柴油是一种可再生和可生 物降解的燃料. 其来源广泛, 如植物油、动物脂肪或者 其他可再生的之类. 目前认为甘油三酯在酶的条件下转 化为生物柴油的方式是最为绿色的. 但是相应的也产生 了一些问题, 如成本高, 原油和废油中的一些杂质会使 得脂肪酶失活以及挥发性有机溶剂的使用等. 2013 年, 该课题组 ${ }^{[31]}$ 报道了应用胆碱基的低共熔溶剂作为溶剂 以大豆油为原料在酶催化条件下制备得到生物柴油的 方法. 该方法采用胆碱基质的低共熔溶剂，克服了成本 高的问题, 而且 低共熔溶剂无毒, 与脂肪酶的生物相 容性好. 结果表明, 最佳的反应条件为 $\mathrm{ChCl}$ /甘油(1：2) 组成的低共熔溶剂与甲醇以 $7: 3$ 的比例混合, 以 Novozym ${ }^{\circledR} 435$ 作为催化剂, 在 $50{ }^{\circ} \mathrm{C}$ 下反应 $24 \mathrm{~h}$, 甘油 三酯的最高产率为 $88 \%$.

\section{5 低共熔溶剂在其他反应中的应用}

2011 年, Azizi 等 ${ }^{[32]}$ 报道了在 $\mathrm{ChCl} /$ 尿素中应用硼氢 化钠化学选择性还原功能化的羧酸衍生物和环氧化合 物(Scheme 7). 具体是在室温条件下, 加入 2 equiv. 的硼 氢化钠, 大多数的醛和酮都可以在 $\mathrm{ChCl} /$ 尿素中不到 5 $\min$ 的时间就可以被还原, 所得相应的醇的产率为 $75 \% \sim 99 \%$. 除此之外，大部分环氧化合物也可在 $\mathrm{ChCl} /$ 尿素中被还原，而相应的醇的产率为 $75 \% \sim 95 \%$.

2012 年 Coulembier 等 ${ }^{[33}$ 报道了应用 1,8 -二氮杂二 环[5.4.0]十一碳-7-烯(DBU)作为作碱性催化剂催化丙交 酯(LA)和 1,3-二氧杂环己烷-2-酩(TMC)共聚的文章. 聚 乳酸是一种更为安全和可生物降解的聚合物, 由于丙交

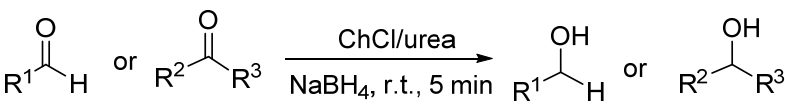

$$
\begin{aligned}
& \stackrel{\mathrm{ChCl} / \text { urea }}{\stackrel{\mathrm{O}}{\mathrm{NaBH}, 60^{\circ} \mathrm{C}, 30 \sim 180 \mathrm{~min}}}
\end{aligned}
$$

图式 7 在低共熔溶剂体系中进行羰基与环氧化合物的还原 反应

Scheme 7 Reduction of carbonyl compounds and epoxides in deep-eutectic solvent

酯在溶解过程中应用了一些具有潜在毒性的有机溶剂, 所以大大地降低了其的环境友好性和使用范围. 但是作 者却发现 1: 1 配比的 LA 和 TMC 可以在 $21.3{ }^{\circ} \mathrm{C}$ 时形 成一种低共熔溶剂(Scheme 8). 在苄醇作为引发剂, DBU 作为催化剂的条件下, 聚乳酸(PLLA)链开始成核, 并且从熔体中结晶，从而限制了 PLLA 的增长. 更为有 趣的是, 当溶剂加热到 $60{ }^{\circ} \mathrm{C}$ 时或者迅速地溶解于二氯 甲烷中时, PLLA 就会与 TMC 相互作用, 并迅速地从熔 体中析出.

近年研究表明, 低共熔溶剂可以作为一种更为安全 与便宜的替代品代替咪唑类离子液体. 其中, 最为典型 的当属 5-着圣甲基糠醛(HMF)的制备. HMF 是一种重要的 有机合成中间体，特别是可以作为一些聚合物和燃料添 加剂的组成部分. 例如, 2009 年 König 等 ${ }^{[34]}$ 报道了在 $\mathrm{ChCl} /$ urea (1：2)组成的低共熔溶剂中由果糖通过酸催 化脱水制备 HMF 的方法. 在此过程中, 使用的果糖是 高浓度的(40\%), 并使用了酸催化剂(10 $\mathrm{mol} \% \mathrm{CrCl}_{2}$, $\mathrm{CrCl}_{3}, \mathrm{FeCl}_{3}, \mathrm{AlCl}_{3}$ 或 Amberlyst 15). 然而 $\mathrm{HMF}$ 的产率 却很低 $(<30 \%)$, 这是由于果糖与尿素的之间存在副反 应. 当应用四甲基脲代替尿素之后，副反应得到抑制， 此时在 $10 \mathrm{~mol} \% \mathrm{FeCl}_{3}$ 的催化条件下 $\mathrm{HNF}$ 的产率高达 $89 \%$ ．但是四甲基脲的应用也带来了两个明显问题：试
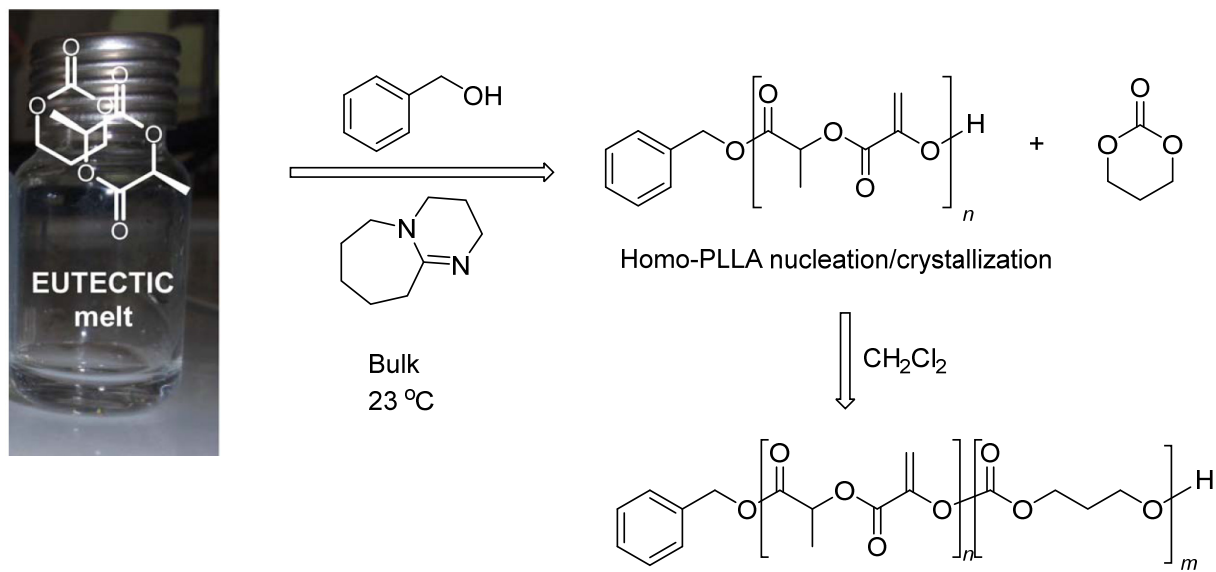

图式 8 以 DBU 作催化剂在 LA-TMC 组成的低共熔溶剂中进行共聚合反应

Scheme 8 Co-polymerization of the LA-TMC eutectic mixture in the presence of DBU as catalyst 
剂毒性和产物分离的问题. 该课题组通过应用碳水化合 物与 $\mathrm{ChCl}$ 共混形成的低共熔溶剂来取代之前的低共熔 溶剂, 从而解决了这些问题. 这种由碳水化合物组成的 高浓度熔体有着低熔点 $\left(<80{ }^{\circ} \mathrm{C}\right)$, 低粘度和低毒性的 特点. 但是, 上述方法存在一个明显缺陷就是在反应过 程中使用了金属催化剂, 一方面使成本增加, 另外给环 境带来一定的污染. Han 等 ${ }^{[35]}$ 报道了在低共熔溶剂中无 需金属氯化物的催化就可制备 HMF 的方法. 作者在 $\mathrm{ChCl} /$ 柠檬酸组成的布朗斯特酸 低共熔溶剂体系中果 糖脱水制备 HMF $\left(80{ }^{\circ} \mathrm{C}, 1 \mathrm{~h}, \mathrm{DES}:\right.$ 果糖 $\left.=5: 1\right)$, 其产 率高达 $76 \%$. 柠檬酸是一种便宜、可再生的羧酸, 其可 以通过农业的废弃物发酵而大规模的制备. 由于 HMF 在 $\mathrm{ChCl}$ /柠檬酸组成的低共熔溶剂中溶解度很低, 但用 乙酸乙酯萃取出 HMF, 其产率仍可达 $91 \%$. 研究表明, 当在循环实验时 HMF 的产率不会随低共熔溶剂使用次 数的增加而有明显的降低. 当 $\mathrm{ChCl} /$ 柠檬酸组成的低共 熔溶剂干燥之后, HMF 又恢复了最初的产率, 更证明了 该种低共熔溶剂的催化稳定性. 随后, Han 等 ${ }^{[36]}$ 将上述 工艺进行了延续, 他们应用菊粉通过降解和脱水来制备 HMF. 结果表明, 当采用 $\mathrm{ChCl} /$ 草酸和 $\mathrm{ChCl} /$ 柠檬酸组成 的低共熔溶剂, HMF 的产率分别为 $56 \%$ 和 $51 \%\left(80{ }^{\circ} \mathrm{C}, 2\right.$ $\mathrm{h}$, 果糖在低共熔溶剂中的浓度为 $16 \mathrm{~mol} \%$ ). 值得注意 的是, 与之前的报道相比较, 在这个过程中加入适量的 水可以增加 HMF 的产率. 在这个系统中, 水作为一种 控制菊粉分解为果糖的关键因素. 所以在制备低共熔溶 剂的过程中要使用含水的草酸和柠檬酸. $\mathrm{ChCl} /$ 草酸组 成的低共熔溶剂成功地回收 6 次后, HMF 产率没有明显 的下降.

与之类似的还有, Vigier 等 ${ }^{[37]}$ 报道了以甜菜碱盐酸 盐(BHC)作为一种可再生的布朗斯特酸与 $\mathrm{ChCl}$ 和水构 成的低共熔溶剂用于制备 HMF 的方法. 在这三组分 ( $\mathrm{ChCl} / \mathrm{BHC} /$ 水) 低共熔溶剂中, $\mathrm{HMF}$ 的产率为 $63 \%$ $\left(130{ }^{\circ} \mathrm{C}\right.$, 果糖的之质量分数为 $\left.40 \%\right)$. 与上述 $\mathrm{Han}$ 等的 工作相比, 当该反应用两相体系(甲基异丁基酮作为萃 取溶剂)时, HMF 的纯品产率高达 $95 \%$ 以上, 相较于单 相体系中 $84 \%$ 的产率, 有着明显的提高. 这也就进一 步证明了该体系有着与传统的咪唑类离子液体相似的 特性. 同样的, 该种三组分低共熔溶剂成功回收 7 次后, 仍然具有催化效果.

另外, 低共熔溶剂也可以应用于酰胺合成方面. 酰 胺在天然产物和合成化学中作为一种重要的链结结构, 其合成方法也繁多, 如羧酸与胺、酰氯与胺、醇与胺等. 2014 年, Nagarkar 等 ${ }^{[38]}$ 报道了应用 $\mathrm{ChCl}$ 基低共熔溶剂 $\left(\mathrm{ChCl} / \mathrm{ZnCl}_{2}=1: 2\right)$ 作为溶剂以醛和腈为原料合成酰胺 的简单方法(Scheme 9). 相较于传统的合成方法, 该方
法更加高效、绿色，在降低成本的同时，更为环保，产率 更高 (高达 98\%).
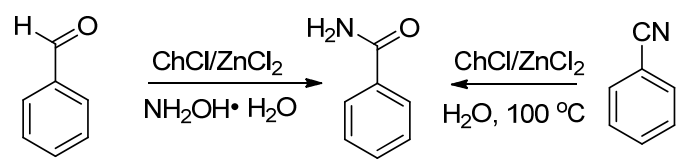

图式 9 在低共熔溶剂体系中制备苯甲酰胺

Scheme 9 Synthesis of benzamide in deep-eutectic solvent

\section{4 展望}

与有机溶剂、离子液体相比, 低共熔溶剂有着明显 的优点：(1)原料价廉易得、制备方便; (2)制备过程绿色 且原子利用率为 $100 \%$; (3)低毒性, 是一种真正的绿色 溶剂. 同时, 低共熔溶剂体系还能实现回收和重复利用, 当体系中产物被有机溶剂萃取后, 旋转蒸发除去萃取、 洗涤过程带进体系的水(低共熔溶剂溶于水), 即可方便 实现对低共熔溶剂的回收. 从已经报导的大部分实验结 果来看, 回收的低共熔溶剂仍然能够保持良好的稳定 性，将其重新用于下一个反应循环后，反应产率降低不 太明显. 除此之外, 从催化化学和有机合成角度来说, 低共熔溶剂有着与咪唑类离子液体类似的溶解性, 在采 用不同的氢键供体和氢键受体时可以配制出不同的低 共熔溶剂，这也就使得低共熔溶剂种类繁多. 同时, 由 于其自身存在有酸碱性, 可以在不外加催化剂的条件下 催化部分反应. 因此, 可以预计，在不远的未来，低共 熔溶剂有望取代价格高昂的离子液体, 成为一种更为绿 色, 更为便宜的溶剂.

\section{References}

[1] Hallett, J. P.; Welton, T. Chem. Rev. 2011, 111, 3508.

[2] Walsh, D. A.; Lovelock, K. R. J.; Licence, P. Chem. Soc. Rev. 2010, $39,418$.

[3] Bideau, J. L.; Viau, L.; Vioux, A. Chem. Soc. Rev. 2011, 40, 907.

[4] Xiong, X. Q.; Yi, C.; Han, Q.; Shi, L. Chin. J. Catal. 2015, 36, 237.

[5] Plechkova, N. V.; Seddon, K. R. Chem. Soc. Rev. 2008, 37, 123.

[6] Zhang, Q. H.; Vigier, K. D. O.; Royer, S.; Jérôme, F. Chem. Soc. Rev. 2012, 41, 7108.

[7] Ruß, C.; König, B. Green Chem. 2012, 14, 2969.

[8] Smith, E. L.; Abbott, A. P.; Ryder, K. S. Chem. Rev. 2014, 114, 11060 .

[9] Abboott, A. P.; Capper, G.; Davies, D. L.; Rasheed, R. K.; Tambyrajah, V. Chem. Commun. 2003, 70.

[10] Imperato, G.; Höger, S.; Lenoir, D.; König, B. Green Chem. 2006, $8,1051$.

[11] Imperato, G.; Eibler, E.; Niedermaier, J.; König, B. Chem. Commun. 2005, 1170.

[12] Ilgen, F.; König, B. Green Chem. 2009, 11, 848.

[13] Singh, B. S.; Lobo, H. R.; Pinjari, D. V.; Jarag, K. J.; Pandit, A. B.; Shankarling, G. S. Ultrason. Sonochem. 2013, 20, 633.

[14] Zhang, Z.-H.; Zhang, X.-N.; Mo, L.-P.; Li, Y.-X.; Ma, F.-P. Green Chem. 2012, 14, 1502.

[15] Chen, Z.; Zhou, B.; Cai, H.; Zou, X. Green Chem. 2009, 11, 275. 
[16] Phadtare, S. B.; Shankarling, G. S. Green Chem. 2010, 12, 458.

[17] Singh,B.; Lobo, H.; Shankarling, G. Catal. Lett. 2011, 141, 178.

[18] De Santi, V.; Cardellini, F.; Brinchi, L.; Germani, R. Tetrahedron Lett. 2012, 53, 5151.

[19] Imperato, G.; Vasold, R.; König, B. Adv. Synth. Catal. 2006, 348, 2243.

[20] Wang, A. L.; Xing, P. F.; Zheng, X. L.; Cao, H. Y.; Yang, G.; Zheng, X. F. RSC Adv. 2015, 5, 59022.

[21] Pawar, P. M.; Jarag, K. J.; Shankarling, G. S. Green Chem. 2011, 13, 2130.

[22] Singh, B. S.; Lobo, H. R.; Shankarling, G. S. Catal. Commun. 2012, 2470 .

[23] Sonawane, Y. A.; Phadtare, S. B.; Borse, B. N.; Jagtap, A. R.; Shankarling, G. S. Org. Lett. 2010, 12, 1456.

[24] Azizi, N.; Gholibeglo, E. RSC Adv. 2012, 2, 7413.

[25] Azizi, N.; Rahimi, Z.; Alipour, M. RSC Adv. 2015, 5, 61191.

[26] Iha, R. K.; Wooley, K. L.; Nystrom, A. M.; Burke, D. J.; Kade, M. J.; Hawker, C. J. Chem. Rev. 2009, 109, 5620.

[27] Azizi, N.; Mariami, M.; Edrisi, M. Dyes Pigm. 2014, 100, 215.
[28] Keshavarzipour, F.; Tavakol, H. Catal. Lett. 2015, 145, 1062.

[29] Gorke, J. T.; Srienc F.; Kazlauskas, R. J. Chem. Commun. 2008, 1235.

[30] Zhao, H.; Baker, G. A.; Holmes, S. Org. Biomol. Chem. 2011, 9, 1908.

[31] Zhao, H. Zhang, C.; Crittle, T. D. J.Mol. Catal. B: Enzym. 2013, 85.

[32] Azizi, N.; Batebi, E.; Bagherpour, S.; Ghafuri, H. RSC Adv. 2012, 2, 2289.

[33] Coulembier, O.; Lemaur, V.; Josse, T.; Minoia, A.; Cornil, J.; Dubois, P. Chem. Sci. 2012, 3, 723.

[34] Ilgen, F.; Ott, D.; Kralisch, D.; Reil, C.; Palmbergera, A.; König, B. Green Chem. 2009, 11, 1948.

[35] Hu, S.; Zhang, Z.; Zhou, Y.; Han, B.; Fan, H.; Li, W.; Song, J.; Xie, Y. Green Chem. 2008, 10, 1280.

[36] Hu, S.; Zhang, Z.; Zhou, Y.; Song, J.; Fan, H.; Han, B. Green Chem. 2009, 11, 873 .

[37] De Oliveira Vigier, K.; Benguerba, A.; Barrault, J.; Jérôme, F. Green Chem. 2012, 14, 285.

[38] Patil, U. B.; Singh, A. S.; Nagarkar, J. M. RSC Adv. 2014, 4, 1102. 